The Astrophysical Journal, $199:$ L19-L24, 1975 July 1

(c) 1975. The American Astronomical Society. All rights reserved. Printed in U.S.A.

\title{
RED GIANTS AND SUPERGIANTS WITH DEGENERATE NEUTRON CORES*
}

\author{
Kip S. THORNE \\ California Institute of Technology \\ AND \\ ANNA N. ŻYTKOW \\ Institute of Astronomy, Polish Academy of Sciences, Warsaw; Institute of Astronomy, University of Cambridge, England; \\ and California Institute of Technology \\ Received 1974 December 9; revised 1975 March 17
}

\begin{abstract}
A new type of stellar model is constructed. It is related to neutron stars as ordinary red giants are related to white dwarfs. Its external appearance is similar to that of an ordinary $\mathrm{M}$ supergiant, but its evolutionary lifetime is 10 times longer.

Our models are constrained to be relativistic but nonrotating, and to contain a degenerate neutron core of mass $1 M_{\odot}$ and radius $10 \mathrm{~km}$, surrounded by a nondegenerate, massive, diffuse envelope. The core and envelope turn out to be separated by a thin $(\sim 40 \mathrm{~m})$ energy-generation layer. The envelope convects from this layer all the way out to the photosphere. The effective temperatures and radii are $\sim 2700 \mathrm{~K}$ and $\sim 1000 R_{\odot}$. Within a fairly narrow range of effective temperatures and radii, two families of models were found: "red giants" and "red supergiants" with luminosities and masses less than and greater than $\sim 65,000 L_{\odot}$ and $\sim 10 M_{\odot}$, respectively. The luminosity of a giant comes 97 percent from gravitational contraction and 3 percent from nuclear burning. That of a supergiant is 5 percent from gravitational contraction and 95 percent from hydrogen burning by nonequilibrium, hot $\mathrm{CNO}$ reactions. The $\mathrm{CNO}$ reaction products are convected directly from the hydrogen-burning shell out to the photosphere of the supergiant, where they should be observable.
\end{abstract}

Subject headings: interiors, stellar - neutron stars - stellar evolution

\section{COMPUTER-GENERATED MODELS}

In this Letter we present the first results of computations of spherically symmetric stellar models with degenerate neutron cores. The computer program that generates these models divides the star into an "outer region" (static part of the envelope) extending in to the point where

$$
U / c^{2}+P / \rho c^{2}+G M_{r} / r c^{2}=3 \times 10^{-4} ;
$$

a "middle region" (stationary inflowing part of the envelope plus the neutron core's "halo") extending from the point defined by equation (1) in to a density of $\rho=3 \times 10^{8} \mathrm{~g} \mathrm{~cm}^{-3}$; and an "inner region" (core) with $\rho>3 \times 10^{8}$ $\mathrm{g} \mathrm{cm}^{-3}$.

The model for the outer region is generated using a slightly modified version of Paczynski's (1969) program for calculating static stellar envelopes with extended atmospheres. This program includes (i) Newtonian equations of stellar structure with luminosity constant throughout and with a mixing-length formalism for convection; (ii) an opacity table for composition $X=0.7, Z=0.03$ (Paczynski 1970), interpolated from the Cox-Stewart (1968) opacities and augmented by an approximation to Auman's (1967) $\mathrm{H}_{2} \mathrm{O}$ opacity; and (iii) an equation of state including the effects of ionization of $\mathrm{H}$ and $\mathrm{He}$, dissociation of $\mathrm{H}_{2}$, free electrons, and radiation.

The model for the middle region is generated by our own computer routine, which is a general-relativistic version of Paczynski's (1970) method for constructing stellar models with gravitational contraction and with very thin shell sources (Paczynski's method is well justified here because the mass in the contracting region is only $\sim 10^{-10} M_{\odot}$ ). In the energy conservation equation we include (i) energy generation by burning of hydrogen (CNO cycle), helium $(3 \alpha)$, and carbon $(\mathrm{C}+\mathrm{C})$; (ii) energy release by gravitational contraction-assuming a constant rate of mass inflow; (iii) neutrino energy losses; and (iv) gravitational redshift of outflowing luminosity. Convection is treated by a mixing-length formalism which incorporates the general-relativistic Schwarzschild criterion (cf. Thorne 1966). Our opacity includes electron and positron scattering (with special-relativistic corrections to the Thomson cross section) and heat conduction by degenerate electrons (Paczynski's analytic fit to the tables of Canuto 1970). In the equation of state we include the effects of radiation, completely ionized nuclei, ionization electrons (taking account of de-

* Supported in part by the National Science Foundation [MPS75-01398, GP-28027] at Caltech. 
generacy and partial degeneracy by the method of Eggleton, Faulkner, and Flannery 1973), and electron-positron pairs in the nonrelativistic, nondegenerate approximation.

The inner region is taken to be a standard neutron-star model. Trial integrations, beginning in the interior of an isothermal neutron core and going out into our model envelopes, show that the thermal conductivity at $3 \times 10^{8} \leq \rho$ $\leq 3 \times 10^{11}$ can support only a negligible amount of heat flow $\left(\leq 100 L_{\odot}\right) .{ }^{1}$ Thus the thermal structure of the inner region $\left(\rho>3 \times 10^{8}\right)$ has most probably no influence on the rest of the star. Instead of analyzing the inner region in detail, we impose inner boundary conditions

$$
L_{r}=0, \quad r=R_{\text {core }}, \quad M_{r}=M_{\text {core }}, \quad T=T_{0} \quad \text { at } \quad \rho=3 \times 10^{8} \mathrm{~g} \mathrm{~cm}^{-3},
$$

on our middle-region integrations. Here $T_{0}$ is an unknown temperature to be determined by integrations of the equations of stellar structure.

At the outer boundary of our model we impose the usual photospheric thermal condition $L=4 \pi \sigma\left(r_{\text {phot }}\right)^{2}\left(T_{\text {phot }}\right)^{4}$. We start our integrations far outside the photosphere using the method of Paczynski (1969).

To obtain a stellar model with constant rate of mass inflow $\dot{M}$ from envelope to core, we choose a core mass, $M_{\text {core }}$, a core radius, $R_{\text {core }}$, a total mass, $M$, and a chemical composition $X_{e}, Z_{e}$ for the envelope. We then integrate our equations inward through the star, iterating the total luminosity $L$, photospheric temperature $T_{\text {phot }}$, and mass inflow rate $\dot{M}$ until they produce the desired $M_{\text {core }}, R_{\text {core }}, M$, and inner boundary conditions (2).

Table 1 and Figure 1 show details of models with $M_{\text {core }}=1 M_{\odot}, R_{\text {core }}=10 \mathrm{~km}, X_{e}=0.70, Z_{e}=0.03$, and with total mass $M$ between $2 M_{\odot}$ and $25 M_{\odot}$. Notice that there is a mass and luminosity gap: we could not construct models with $9.2 M_{\odot}<M<11.3 M_{\odot}$, corresponding to $61,300 L_{\odot}<L<70,600 L_{\odot}$. The models below this gap we shall call "giants"; those above we shall call "supergiants."

Each model consists of three qualitatively different regions (not the same as the "inner," "middle," and "outer" regions used in the computational method): a degenerate "core" $\left(\rho \gtrsim 10^{6}\right)$; a 40-meter thick "halo" surrounding the core-a "halo" that is nondegenerate, nonconvective, and nearly isothermal, and in which nearly all the gravitational energy release occurs; and a fully convective "envelope," extending from the outer edge of the "halo" $(r \approx 10.04 \mathrm{~km})$ all the way out to the star's atmosphere $\left(r \sim 1000 R_{\odot}\right)$. The interface between halo and envelope we call the model's "knee" because of the sharp, kneelike bend in the temperature-density plot there (Fig. 1).

In the giant models nuclear burning occurs entirely in the halo and core, and accounts for only 3 percent of the energy release (compared to 97 percent from gravitational contraction). In the supergiants the hydrogen-burning shell extends from the halo, through the knee, into the envelope. The burning of envelope hydrogen, as convection circulates it through the shell, accounts for 95 percent of the supergiant luminosity. The remaining 5 percent comes primarily from gravitational contraction.

\footnotetext{
${ }^{1}$ This conclusion might change if the "standard" conductivity (Canuto 1970; Flowers and Itoh 1974) were replaced by that of a highly magnetized, degenerate electron gas.
}

TABLE 1

Stellar Models with a $1 M_{\odot}, 10$-Kilometer Core, and with $X_{e}=0.70, Y_{e}=0.27, Z_{e}=0.03$ *

\begin{tabular}{|c|c|c|c|c|c|c|c|c|}
\hline $\begin{array}{l}\text { Type of } \\
\text { Model }\end{array}$ & $\begin{array}{l}\text { Total } \\
\text { Mass } \\
\left(M_{\odot}\right)\end{array}$ & $\begin{array}{c}\text { Total } \\
\text { Luminosity } \\
\left(L_{\odot}\right)\end{array}$ & $\begin{array}{c}\text { Effective } \\
\text { Temperature } \\
(\mathrm{K})\end{array}$ & $\begin{array}{l}\text { Photospheric } \\
\text { Radius } \\
\left(R_{\odot}\right)\end{array}$ & $\begin{array}{c}\text { Contraction } \\
\text { Rate } \\
\left(10^{-8} M_{\odot} \mathrm{yr}^{-1}\right)\end{array}$ & $\begin{array}{c}\text { Temperature } \\
\text { at Knee } \\
\left(10^{8} \mathrm{~K}\right)\end{array}$ & $L_{\text {nuc }} / L$ & $\begin{array}{c}\text { Dynamically, } \\
\text { Adiabatically } \\
\text { Stable? }\end{array}$ \\
\hline $\begin{array}{l}\mathrm{R}, \alpha=1 \ldots \ldots \ldots \\
\mathrm{R}, \alpha=1 \ldots \ldots \ldots \\
\mathrm{R}, \alpha=1 \ldots \ldots \ldots \\
\mathrm{R}, \alpha=1 \ldots \ldots \ldots \ldots \\
\mathrm{R}, \alpha=1 \ldots \ldots \ldots \ldots\end{array}$ & $\begin{array}{l}2.0 \\
3.0 \\
5.0 \\
8.0 \\
9.0\end{array}$ & $\begin{array}{l}38220 \\
38430 \\
41740 \\
55680 \\
60380\end{array}$ & $\begin{array}{l}2670 \\
2581 \\
2667 \\
2787 \\
2819\end{array}$ & $\begin{array}{r}917 \\
984 \\
961 \\
1016 \\
1034\end{array}$ & $\begin{array}{l}1.575 \\
1.584 \\
1.720 \\
2.281 \\
2.478\end{array}$ & $\begin{array}{l}1.26 \\
1.30 \\
1.77 \\
3.64 \\
4.37\end{array}$ & $\begin{array}{l}0.030 \\
0.030 \\
0.030 \\
0.030 \\
0.030\end{array}$ & $\begin{array}{l}\text { NO } \\
\text { NO } \\
\text { YES } \\
\text { YES } \\
\text { YES }\end{array}$ \\
\hline \multicolumn{9}{|c|}{ MASS GAP } \\
\hline $\begin{array}{l}\mathrm{R}, \alpha=1 \ldots \ldots \ldots \ldots \\
\mathrm{R}, \alpha=1 \ldots \ldots \ldots \ldots \\
\mathrm{R}, \alpha=1 \ldots \ldots \ldots \\
\mathrm{R}, \alpha=1 \ldots \ldots \ldots \ldots \\
\mathrm{R}, \alpha=1 \ldots \ldots \ldots \ldots\end{array}$ & $\begin{array}{l}11.5 \\
12.0 \\
16.0 \\
20.0 \\
25.0\end{array}$ & $\begin{array}{r}71360 \\
73530 \\
90490 \\
105600 \\
123300\end{array}$ & $\begin{array}{l}2890 \\
2902 \\
2984 \\
3050 \\
3117\end{array}$ & $\begin{array}{l}1070 \\
1077 \\
1130 \\
1168 \\
1209\end{array}$ & $\begin{array}{l}0.167 \\
0.166 \\
0.156 \\
0.150 \\
0.144\end{array}$ & $\begin{array}{l}9.48 \\
9.51 \\
9.70 \\
9.82 \\
9.94\end{array}$ & $\begin{array}{l}0.947 \\
0.949 \\
0.961 \\
0.968 \\
0.974\end{array}$ & $\begin{array}{l}\text { YES } \\
\text { YES } \\
\text { YES } \\
\text { YES } \\
\text { YES }\end{array}$ \\
\hline $\begin{array}{l}\mathrm{N}, \alpha=1 \ldots \ldots \ldots \\
\mathrm{R}, \alpha=0.5 \ldots \ldots \ldots \\
\mathrm{R}, \alpha=1.5 \ldots \ldots \ldots\end{array}$ & $\begin{array}{l}5.0 \\
5.0 \\
5.0\end{array}$ & $\begin{array}{l}41770 \\
38180 \\
\text { IN MASS }\end{array}$ & $\begin{array}{c}2667 \\
2222 \\
\text { GAP; NO }\end{array}$ & $\begin{array}{c}961 \\
1324 \\
\text { MODEL EXI }\end{array}$ & ISTS $\begin{array}{r}1.852 \\
1.573\end{array}$ & $\begin{array}{l}1.06 \\
1.40\end{array}$ & $\begin{array}{l}0.038 \\
0.030\end{array}$ & $\begin{array}{l}\text { YES } \\
\text { YES }\end{array}$ \\
\hline
\end{tabular}

* In column (1), "R" means general relativistic, " $N$ " means Newtonian, and $\alpha=$ (mixing length)/(pressure scale height). In column (8), $L_{\text {nuc }} / L$ is the fraction of the total luminosity produced by nuclear reactions. In all cases neutrino losses are negligible $\left(<L_{\odot}\right)$, so the remaining luminosity $1-L_{\text {nuc }} / L$ is produced by gravitational contraction. The last column refers to stability of the envelope against adiabatic, radial perturbations. 


\section{THE GIANT MODELS: SEMIQUANTITATIVE EXPLANATION OF STRUCTURE}

Consider first the halo of the giant models, beginning at the edge of the core where the plasma becomes nondegenerate, and moving outward. In giant models the layers of nuclear energy release (which lie in the core and at the bottom of the halo) are well separated from the region of gravitational energy release (which is in the outer part of the halo).

Throughout the halo the luminosity is small compared to the "Eddington critical luminosity," at which photon forces on the plasma would counterbalance gravity: $L_{r} \ll L_{r}$ crit, where

$$
\begin{aligned}
L_{r}^{\text {crit }} & =\left(4 \pi c G M_{r} / \kappa\right) \cdot[1+(\text { general-relativistic corrections } \leq 0.3)] \\
& =\left(0.324 \times 10^{5} L_{\odot}\right)\left(\kappa / 0.4 \mathrm{~cm}^{2} \mathrm{~g}^{-1}\right)^{-1}[1+(\mathrm{GR} \text { corrections })] \sim 10^{5} L_{\odot} .
\end{aligned}
$$

In the second line we have used $M_{r} \approx M_{\text {core }}=1 M_{\odot}$ (valid throughout halo). Consequently, the plasma hardly notices the radiation forces at all; it has the small scale height of a neutron-star atmosphere,

$$
\lambda_{P}=\left|\frac{d r}{d \ln P_{\text {plasma }}}\right| \approx \frac{P_{\text {plasma }} / \rho c^{2}}{\left(G M_{\text {core }} / R_{\text {core }} c^{2}\right)\left(1-L_{r} / L_{r}{ }^{\text {crit }}\right)} R_{\text {core }} \approx(1 \text { meter })\left(\frac{T}{10^{8} \mathrm{~K}}\right) .
$$

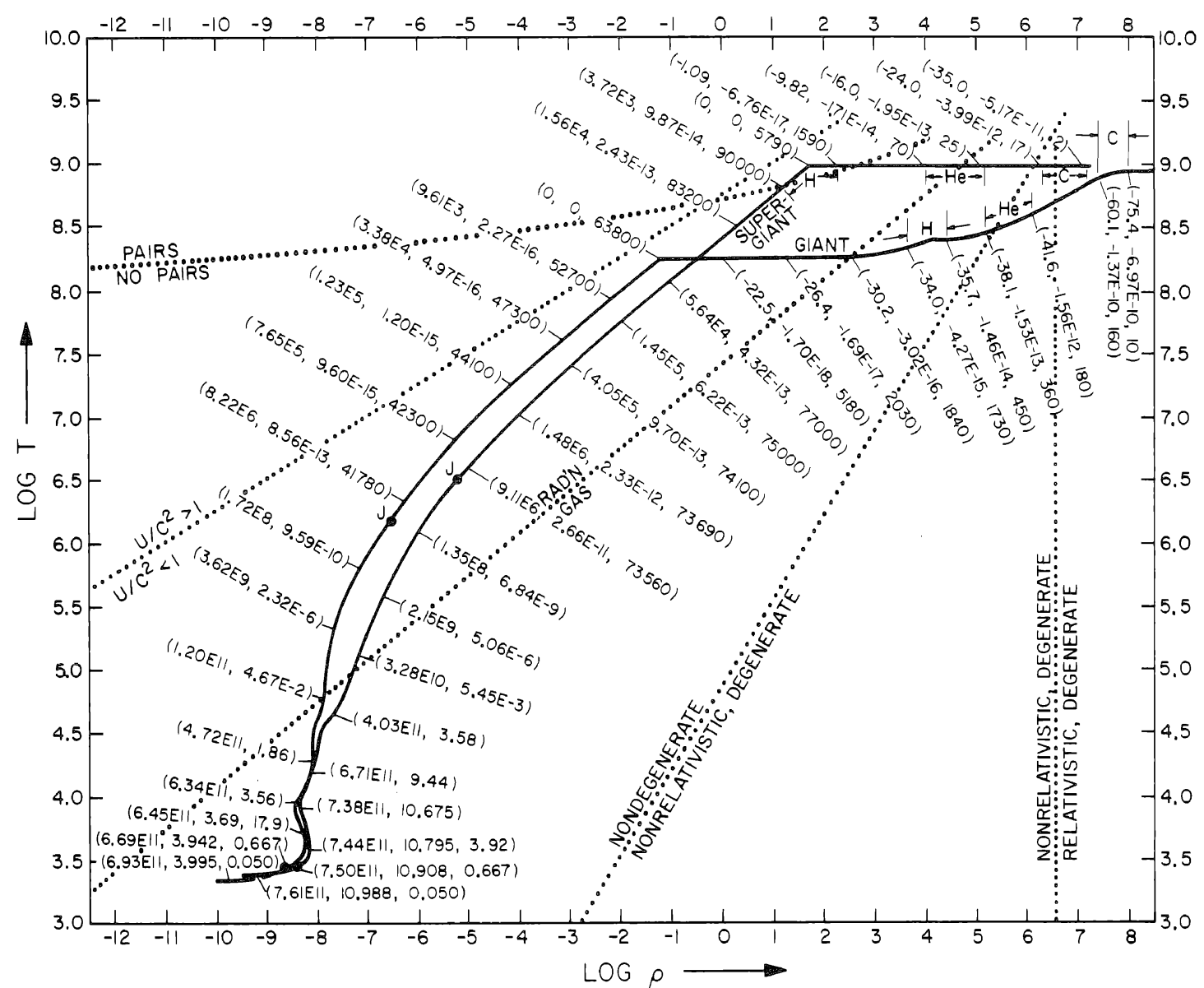

FIG. 1.-Internal structures of a typical "giant" $\left(M=5 M_{\odot}\right)$ and a typical "supergiant" $\left(M=12 M_{\odot}\right)$ selected from the models of Table 1. Temperature $T$ is plotted vertically; density of rest mass, horizontally. Radiation pressure equals gas pressure along the dotted line marked RAD'N-GAS; the density of pairs equals the density of ionization electrons along the dotted line PAIRS-NO PAIRS. The parametrized solid lines are the two models. On these lines the nuclear-burning shells are marked $\mathrm{H}, \mathrm{He}$, and $\mathrm{C}$. The dots $\mathrm{J}$ are the join points between the Paczynski-type static envelope and our contracting envelope. Inside the join point the three parameters along each curve are (i) total distance (radial Schwarzschild coordinate $\Delta r$ ) above the "knee" of the star, measured in meters; (ii) total mass-energy $\Delta M_{r}$ above the "knee," measured in solar masses; (iii) locally measured luminosity $L_{r}$, in units of $L_{\odot}$. (The "knee" is the sharp bend in the temperature-density plot at $T \approx 10^{8}$ or $10^{9} \mathrm{~K}$.) Outside the join point $\mathrm{J}$ the two parameters are $\Delta r$ and $\Delta M_{r}$, augmented by optical depth $\tau$ near the surface. The photosphere $(\tau=0.667)$ is marked by a large dot. The envelopes are continuously convective from the photosphere to the knee. At the knee, convection ceases. 
The small luminosity also guarantees that the temperature gradient (which is proportional to $L_{r} / L_{r}$ crit) is small:

$$
\begin{gathered}
\frac{d \ln T}{d \ln \rho}=\frac{1}{4 \gamma_{L} / \gamma_{\theta}-1} \ll 1 ; \quad \gamma_{L} \equiv \frac{\beta_{L}}{1-\beta_{L}} \equiv \frac{L_{r}^{\text {crit }}}{L_{r}}-1 \gg 1 ; \quad \gamma_{g} \equiv \frac{\beta_{\theta}}{1-\beta_{\theta}} \equiv \frac{P_{\text {plasma }}}{P_{\text {radiation }}} ; \\
P_{\text {plasma }}=(\Re / \mu) \rho T ; \quad P_{\text {radiation }}=\frac{1}{3} a T^{4} .
\end{gathered}
$$

(Note that $\gamma_{g} \ll 1 \ll \gamma_{L}$ in the outer part of the halo. It turns out that in the inner part of the halo, if one ignores the contributions of nuclear burning to $L_{r}$, then $1 \ll \gamma_{g} \ll \gamma_{L}$.)

As the plasma contracts inward through this isothermal, small-scale-height, "neutron-star atmosphere" (halo), it produces luminosity by two processes: nuclear burning [(total energy release per gram) $\approx\left(0.007 c^{2}\right) X_{e} \approx 0.005 c^{2}$, and nearly isothermal compression [(total energy release per gram) $\approx$ (total gravitational potential energy stored up as heat when the gas was contracting adiabatically through the convective envelope) $\left.\sim G M_{\text {core }} / R_{\text {core }} \approx 0.14 c^{2}\right]$. Since the nuclear energy release is very small compared to the compressional energy release, the outward increase of luminosity occurs primarily in the outer parts of the halo. There the equation of energy conservation gives

$$
L_{r} \approx \dot{M}\left[\left(U+\frac{P}{\rho}-\frac{G M_{r}}{r}\right)_{r}-\left(U+\frac{P}{\rho}-\frac{G M_{r}}{r}\right)_{\text {bottom of halo }}\right] \approx \dot{M} c^{2}\left(\frac{4}{3} \frac{a T^{4}}{\rho c^{2}}\right) \sim 0.14 \dot{M} c^{2} \text { at the knee . }
$$

Since $L_{r} \propto 1 / \rho, 90$ percent of the halo luminosity is produced in the first decade of density rise $\left(\rho_{\text {knee }}<\rho<10 \rho_{\text {knee }}\right)$. When $L_{r} / L_{r}$ crit has risen to within $\gamma_{g} \sim 10^{-4}$ of unity the temperature gradient becomes nearly adiabatic $(d \ln T /$ $d \ln \rho \approx \frac{1}{3}$, cf. eq. [5]), the plasma contraction is no longer able to release energy, and the scale height has increased from $\lambda_{P} \sim 1$ meter to $\lambda_{P} \sim r \sim 10 \mathrm{~km}$. The "knee" on the (log $T, \log \rho$ )-diagram (Fig. 1), at which the isothermal halo ends and the convective envelope begins, is extremely sharp:

$$
\left|\Delta L_{r} / L_{r}\right| \sim|\Delta \rho / \rho| \sim|\Delta T / T| \sim|\Delta r / r| \sim \gamma_{g} \approx \beta_{g} \sim 10^{-4}
$$

At the knee the force of outflowing radiation on the plasma must counterbalance the enormous pull of gravity; thus there $L_{r} / L_{r}{ }^{\text {crit }}=1$. As one moves outward from the knee, $L_{r}$ remains nearly constant, but $L_{r}{ }^{\text {crit }}$ drops-initially (at $T>10^{7} \mathrm{~K}$ ) because of a "turn-off" of special-relativistic corrections to the Compton cross section and of the general-relativistic corrections of equation (3); later (at $T<10^{6} \mathrm{~K}$ ) because free-free and bound-free opacity are becoming important. The drop of $L_{r}^{\text {crit }}$ drives $L_{r} / L_{r}{ }^{\text {crit }}$ above unity. Actually, $L_{r}$ also drops as one moves outward, largely due to redshift of lumincsity but also due to superadiabatic compression of inflowing gas. However, the drop in $L_{r}$ is more than counterbalanced by the drop in $L_{r}{ }^{\text {crit }} ; L_{r} / L_{r}{ }^{\text {crit }}$ remains always greater than 1 , and the envelope remains always convective.

\section{HOW SUPERGIANTS DIFFER FROM GIANTS}

To understand the difference between our supergiants and giants, imagine an observer who moves inward through the envelope toward the halo. He knows that convection will turn off, and the envelope will end, when $L_{r} / L_{r}{ }_{r}^{\text {crit }}$ drops slightly below unity. If our observer is in a giant, he sees $L_{r}{ }_{r}^{\text {crit }}$ rise as he moves inward, due to a turn-on of special relativistic corrections to the Thomson cross section and of general-relativistic effects; ultimately $L_{r}^{\text {crit }}$ gets as large as $L_{r}$, and the envelope ends. In a supergiant $L_{r}$ is typically bigger than in a giant. As a result, our inward-moving observer sees $L_{r}^{\text {crit }}$ rise toward $L_{r}$, but not quite make it because at $T \sim 10^{8.7} \mathrm{~K}$ electron-positron pairs form in profusion, driving $\kappa$ up and $L_{r}^{\text {crit }}$ down. As the observer moves on inward and $L_{r}^{\text {crit }}$ plummets toward zero, there appears to be no hope for an end to the envelope. However, at $T \sim 10^{9.0} \mathrm{~K}$ hydrogen burning turns on in the envelope, driving $L_{r}$ into an even more rapid plummet than that of $L_{r}{ }_{r}^{\text {crit. }}$. The two meet, and the envelope ends.

Note that, since hydrogen convects rapidly into and back out of the burning layer, the rate at which hydrogen burns is much higher than the rate $\dot{M}$ at which gas contracts from the envelope, through the helium- and carbonburning shells, and onto the core. Hence, our supergiants evolve more rapidly due to changing envelope composition than due to growth of the core. Note also that since the luminosities of the supergiants are produced primarily by hydrogen burning, the supergiants have much smaller $\dot{M}$ than the giants.

\section{DEFICIENCIES AND UNCERTAINTIES IN OUR MODELS}

Mixing length.-Our models display very inefficient convection at temperatures $T \leq 10^{5} \mathrm{~K}$, where most of the envelope mass is located. As a result, the models are highly sensitive to the choice of mixing length. Table 1 shows some of the consequences of changing from our assumed value

$$
\alpha \equiv \text { (mixing length)/(pressure scale height) }=1
$$

to $\alpha=0.5$ or 1.5 . Note that the mass gap, normally at $M \sim 10 M_{\odot}$, gets moved down to $M \sim 5 M_{\odot}$ by changing to $\alpha=1.5$.

CNO burning rates.-In computing hydrogen burning we have used standard CNO burning rates (Cox and Giuli 1968). However, our hydrogen burning occurs at such high temperatures $\left(T \sim 10^{8.4}\right.$ to $\left.T \sim 10^{9.0}\right)$ that it should go 
by a hot CNO cycle (Audouze, Truran, and Zimmerman 1973) rather than by the standard CNO cycle. Moreover, a given atomic nucleus spends only a very short time in the hydrogen-burning shell: in "giant" models it contracts through the shell in a few tens of seconds; in "supergiant" models it convects into and back out of the hydrogen shell in a few tenths of a second or less. As a result, there is insufficient time for equilibrium abundances to be set up; and the burning cannot go by equilibrium reaction networks. A more correct treatment of the hydrogen burning will surely not change our giant models much, but it might have a substantial effect on our supergiants.

Convective overshoot.--In reality convection cannot possibly turn off at the knee as sharply as demanded by equation (7). The momentum of in-moving convective cells will drive them into the halo, smearing out the knee. Rough estimates suggest that this convective overshoot will extend from $\rho_{\text {knee }}$ to $\sim 10 \rho_{\text {knee }}$, thereby modifying the structure of the region of greatest gravitational energy release.

Effect of nentral currents on neutrino losses.-Our neutrino losses (which turned out negligible, $<L_{\odot}$ ) were computed ignoring neutral-current processes. J. R. Bond (private communication) has examined the effect of neutralcurrent neutrino losses on our halo and outer core $\left(\rho \gtrsim 3 \times 10^{8}\right.$; the region thermally coupled to the halo). He estimates that the neutral-current neutrino losses do not exceed the normal neutrino losses.

\section{STABILITY}

We have worried about four possible instabilities of these models:

Dynamical instability of the envelope, caused by the low adiabatic index $\left(\Gamma_{1}<4 / 3\right)$ in the regions of hydrogen and helium ionization, where most of the envelope mass resides. The situation here is similar to that in red supergiants with degenerate white dwarf cores (cf. Paczynski and Ziołkowski 1968), since the envelopes there and here are nearly identical. In such envelopes the thermal and hydrodynamical time scales are comparable, so energy transport has a strong influence on the time development of any instability. We have analyzed the stability of our envelopes ignoring energy transport (stability against linearized adiabatic, radial perturbations). Below $M_{\text {envelope }} \sim 2 M_{\odot}$ our envelopes are adiabatically unstable; above there, they are adiabatically stable. This result suggests (see, e.g., Keeley 1970a, $b$, 1974) that a more correct, nonadiabatic analysis may reveal either pulsational or disruptive instabilities for our least massive envelopes; but that our most massive envelopes might be stable against all perturbations, except convective ones.

Thermal instability of the shell sources. Consider a nonconvective shell source with average luminosity and temperature $\bar{L}_{r}$ and $\bar{T}$, and with luminosity and temperature drop across itself of $\Delta L_{r}$ and $\Delta T$. A crude analysis (cf. p. 857 of Schwarzschild and Härm 1965) shows that an average temperature rise of $\delta T$ inside the shell produces the following rate of increase of the shell's internal energy:

$$
\frac{d E_{\text {internal }}}{d t}=\left(\Delta L_{r}\right)\left[\nu-8 \frac{\bar{L}_{r}}{\Delta L_{r}}-\frac{\bar{T}}{\Delta T}\right] \frac{\delta T}{T} .
$$

Here $\nu$ is the temperature exponent of the nuclear burning rate, $\varepsilon \propto T^{\nu}$. The shell sources in the nonconvective halos of our models are extremely isothermal; so isothermal that a positive $\delta T$ is likely to produce a negative $d E_{\text {in ternal }} /$ $d t$. This suggests that the shell sources in the halo may be stable against thermal runaway.

In our supergiant models the convective hydrogen shell source might be protected from thermal runaway by the high speed at which plasma circulates through it.

Instability of the layer of gravitational energy generation. We have begun to study this, but have reached no conclusions.

Runaway neutrino losses, accompanied by an ever-increasing rate of envelope contraction (Ostriker, private communication; cf. Zel'dovich, Ivanova, and Nadyozhin 1972). The temperatures at $\rho \leq 3 \times 10^{8}$ are so low that neutrino losses there are very small $\left(L_{\nu}<L_{\odot}\right)$, and cannot run away. One can show analytically that the low thermal conductivity at $3 \times 10^{8}<\rho<3 \times 10^{11}$ prevents significant heat flow from envelope into core; this should protect the star against runaway core neutrino losses (but see note 1 ).

In summary, our least massive models almost certainly have unstable envelopes; but our more massive models have some hope of being free of fatal instabilities. More detailed stability studies are much needed.

\section{SOME SPECULATIONS}

Undoubtedly the strongest reason to believe that stars with neutron cores exist in nature is the universal law that "everything not forbidden is compulsory" (White 1939). By comparison, any other basis for discussing existence is extremely uncertain.

Nevertheless, it is fun to speculate.

Can such stars ever be formed by the collapse of the degenerate electron core of a "normal" giant or supergiant? Or must such collapse always deposit enough momentum in the envelope to eject it?

Can supercritical accretion onto an initially naked neutron star produce such stars as these? Might such stars be formed by a neutron star in a close binary system spiraling into its companion's interior? Through such processes might many or most neutron stars in close binaries become enshrouded in envelopes and masquerade as "normal" red supergiants for some 100 million years? 
Matter processed by the burning shells and added to the core of a giant model raises the core mass above the Oppenheimer-Volkov limit within

$$
t_{\text {ife }} \sim M_{\odot} c^{2}\left(G M_{\text {core }} / R_{\text {core }} c^{2}\right) / L \sim M_{\odot} c^{2} /\left(3 \times 10^{5} L_{\odot}\right) \sim 10^{8} \text { years } .
$$

What will happen to the envelope when the core then collapses to form a black hole?

In our supergiant models convection will carry the products of nonequilibrium, hot-CNO hydrogen burning directly from the hydrogen shell out to the photosphere. Hence, the photosphere should exhibit very peculiar relative abundances of various isotopes of carbon, nitrogen, oxygen, fluorine, . . . Might some of the observed peculiar-abundance supergiants be such stars?

In future papers in the Astrophysical Journal we shall present further details of our models and their stability, and of the mass gap between giants and supergiants; and we shall discuss the analogous problem of stars with black-hole cores.

We are deeply indebted to Bohdan Paczynski for suggesting this research problem, for many helpful discussions during its execution, and for making available to us his envelope integration routine. We also gratefully acknowledge valuable discussions with Richard Bond, Wojciech Dziembowski, Douglas Eardley, Douglas Keeley, Jeremiah P. Ostriker, and Martin Schwarzschild, as well as valuable assistance in numerical work from Barbara Zimmerman. For hospitality while this work was underway we thank the Institute of Astronomy in Cambridge, England, and the staff of the Astronomical Observatory in Ostrowik, Poland. Some of K.S.T.'s living expenses were kindly provided by NSF grant GF-36217 to the Institute of Theoretical Physics of Warsaw University, Poland.

\section{REFERENCES}

Audouze, J., Truran, J. W., and Zimmerman, B. A. 1973, Ap. J., $184,493$.

Auman, J., Jr. 1967, Ap. J. Suppl. No. 127, 14, 171.

Canuto, V. 1970, Ap.J., 159, 641.

Cox, A. N., and Stewart, J. N. 1968, Astr. Council, Acad. of Sciences, USSR Sci. Information, 15, 1.

Cox, J. P., and Giuli, R. T. 1968, Principles of Stellar Structure (New York: Gordon \& Breach).

Eggleton, P., Faulkner, J., and Flannery, B. P. 1973, Astr. and Ap., 23, 325.

Flowers, E., and Itoh, N. 1974, preprint from New York University.

Keeley, D. A. 1970a, unpublished Ph.D. thesis, California Institute of Technology.

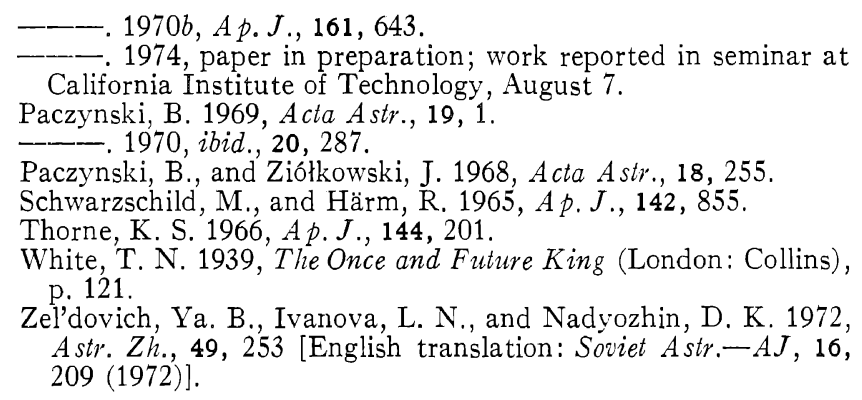

- 1974, paper in preparation; work reported in seminar at California Institute of Technology, August 7.

1970, ibid., 20, 287.

Paczynski, B., and Ziółkowski, J. 1968, Acta Astr., 18, 255.

$, 142,855$

p. 121

A str. $Z$ h 209 (1972)]

Kip S. Thorne and Anna N. Żytkow: Kellogg Radiation Lab. 106-38, California Institute of Technology, Pasadena, CA 91125 